\title{
Compromiso del sistema nervioso central por Eikenella corrodens. Revisión de la literatura médica a propósito de un caso clínico
}

\author{
Commitment of the central nervous system by Eikenella corrodens. \\ Literature review about a clinical case
}

Martín Lasso', Cristóbal Alegría², Andrés Aquevedo² y Sebastián Barría ${ }^{3}$

\begin{abstract}
'Unidad de Infectología Complejo Asistencial "Dr. Sotero Del Rio" y Departamento de Enfermedades Infecciosas, Pontificia Universidad Católica de Chile.
2Unidad de Paciente Critico Complejo Asistencial "Dr. Sótero Del Rio".

${ }^{3}$ Programa de Formación de Subespecialidad Enfermedades Infecciosas, Pontificia Universidad Católica de Chile.
\end{abstract}

Los autores declaran no tener conflictos de interés.

El presente trabajo no cuenta con fuente de financiamiento.

Recibido: 9 de febrero de 2020 / Aceptado: 11 de enero de 2021

\section{Resumen}

Se presenta el caso de un paciente de 22 años sin antecedentes mórbidos que desarrolló un absceso cerebral secundario a una endocarditis por Eikenella corrodens. El diagnóstico se estableció mediante la detección por reacción de polimerasa en cadena universal en el líquido de la colección cerebral. La ecocardiografía transesofágica confirmó vegetaciones en la válvula mitral. Requirió la colocación de una derivación ventricular externa por vaciamiento del absceso a ventrículos cerebrales e hidrocefalia secundaria. Recibió 80 días de tratamiento antibacteriano efectivo. Su evolución fue favorable, con resolución completa de la infección verificada con imágenes y ecocardiografía de control. El seguimiento a los siete meses por una disfunción de válvula de drenaje ventrículo-peritoneal no demostró infección.

Palabras clave: Eikenella corrodens; HACEK; absceso cerebral; endocarditis.

\section{Introducción}

$E$ ikenella corrodens es un bacilo gramnegativo habitante de la microbiota orofaríngea y respiratoria alta. Es un agente oportunista, que causa en forma infrecuente infecciones principalmente en cabeza, cuello y válvulas cardíacas ${ }^{1}$. Pertenece al grupo HACEK (Haemophilus aprophilus y paraprophilus, Actinobacillus actinomycete-

\section{Abstract}

We present the case of a 22-year-old patient with no morbid history who developed a brain abscess secondary to endocarditis due to Eikenella corrodens. The diagnosis was established by detecting the microorganism by universal polymerase chain reaction in fluid from the brain collection. Trans-esophageal echocardiogram study confirmed vegetations in the mitral valve. External ventricular shunt placement was required due to emptying of abscess to brain ventricles and secondary hydrocephalus. He received 80 days of effective antibiotic treatment and his evolution was favorable with complete resolution of his infection verified with images and echocardiogram. Follow-up at seven months later due to ventricule-peritoneal drainage valve dysfunction did not confirmed infection.

Key words: Eikenella corrodens; HACEK; brain abscess; endocarditis.

mcomitans, Cardiobacterium hominis, E. corrodens y Kingella kingae) dentro del cual E. corrodens es el integrante menos frecuente como causa de endocarditis ${ }^{2}$. Como agente etiológico de abscesos cerebrales solo existen reportes anecdóticos en la literatura médica.

Presentamos el caso clínico de un paciente joven sin factores de riesgo que desarrolló un absceso cerebral por E. corrodens, diagnosticándose tardíamente una endocarditis. 


\section{- vil caso Clínico}

\section{Caso clínico}

Varón de 22 años, procedente de zona urbana, sin antecedentes mórbidos conocidos, con una historia de cefalea de dos meses de evolución. Por aparición de compromiso de conciencia, fiebre, náuseas y vómitos fue llevado al servicio de urgencia de nuestro hospital. Ingresó en malas condiciones generales, con compromiso de conciencia cuantitativo rápidamente progresivo (desde puntaje en la escala de Glasgow de 13 a 6/15) por lo que requirió intubación orotraqueal para protección de la vía aerea. Se realizó una tomografía computarizada (TC) de cerebro sin contraste, que evidenció una lesión expansiva fronto-insular derecha, de aspecto heterogéneo, con edema perilesional de la sustancia blanca adyacente, que deformaba el contorno del ventrículo lateral.

Ingresó a la unidad de paciente crítico (UPC) con diagnóstico de un probable tumor cerebral paratalámico derecho y síndrome de hipertensión intracraneana, motivo por el cual recibió sedación, dexametasona, solución

Figura 1. Placa eritematosa circular 3-4 cm de diámetro con múltiples pústulas foliculares confluentes en occipucio compatible con tinea capitis.
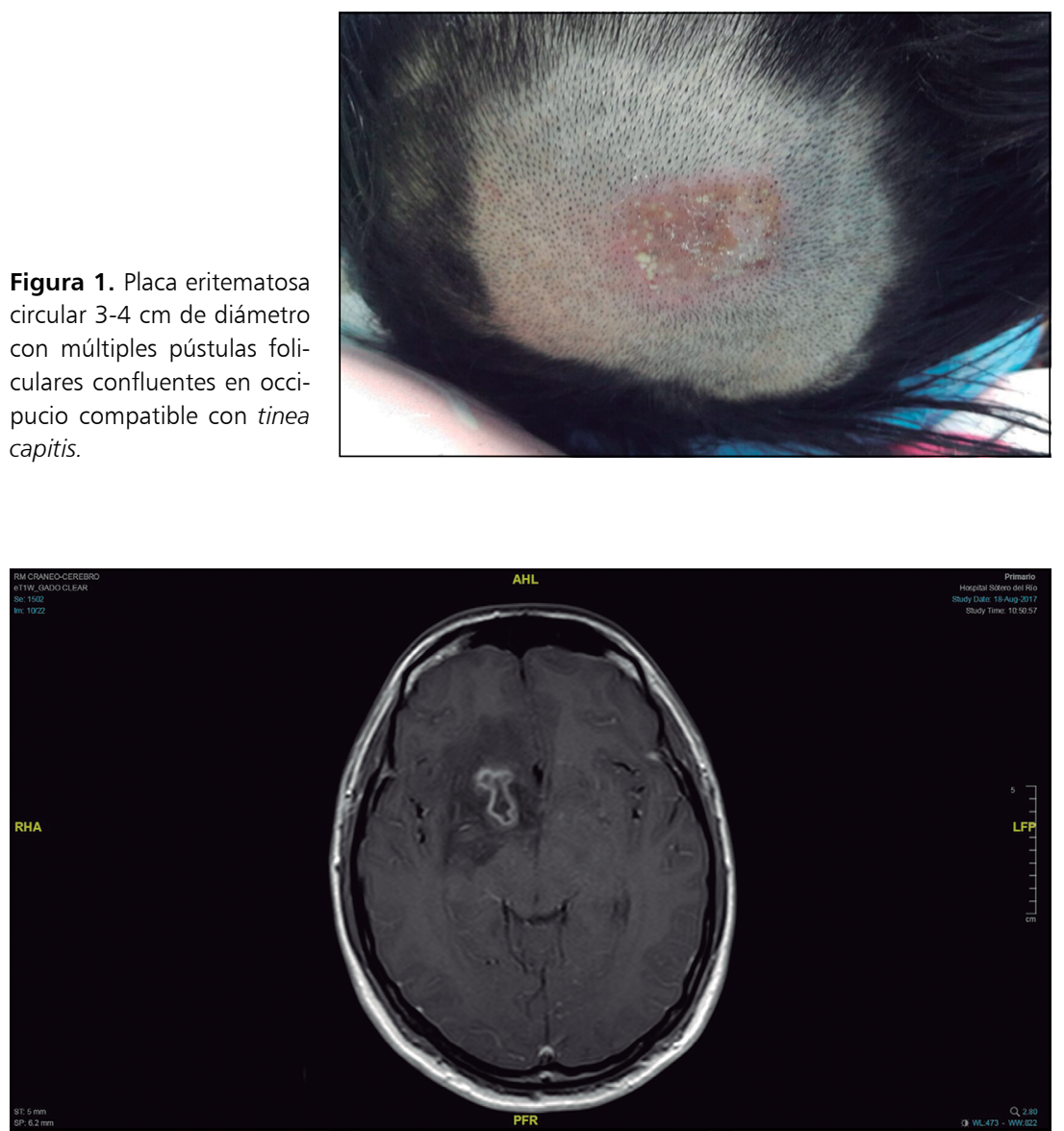

Figura 2. RM cerebral con absceso cerebral frontal derecho de $3 \mathrm{~cm}$ : con captación de contraste (eT1W: gadolinio) tomado $48 \mathrm{~h}$ después del ingreso del paciente. hipertónica y se conectó a ventilación mecánica invasiva (VMI). Al examen físico destacaba una placa eritematosa circular de 3-4 cm de diámetro con múltiples pústulas foliculares confluentes y una zona erosiva periférica compatible con una tinea capitis sobreinfectada por bacterias y cuyo estudio microbiológico resultó negativo (Figura 1).

Por sospecha de una lesión intra-cerebral neoplásica se realizó una TC de tórax, abdomen y pelvis en que no se evidenciaron lesiones primarias. Entre los exámenes de laboratorio destacó un hemograma con leucocitosis de 18.130 céls $/ \mathrm{mm}^{3}$ (92\% PMN) y una PCR de $118 \mathrm{mg} / \mathrm{l}$. La prueba de ELISA para VIH resultó negativa. Se realizó una resonancia magnética (RM) de cerebro que mostró múltiples zonas de aumento de señal en el parénquima supra e infratentorial, con una lesión dominante frontal derecha de aspecto inflamatoria-infecciosa de $3 \mathrm{~cm}$ de diámetro, con captación de gadolinio compatible con un absceso (Figura 2).

El paciente evolucionó febril por lo que se suspendió dexametasona y se inició tratamiento antimicrobiano con ceftriaxona i.v. $2 \mathrm{~g}$ cada $12 \mathrm{~h}$, metronidazol i.v. $500 \mathrm{mg}$ cada $6 \mathrm{~h}$ y vancomicina i.v. $750 \mathrm{mg}$ cada $6 \mathrm{~h}$, previa toma de hemocultivos.

El estudio de LCR mostró un líquido turbio, con proteínas $0,96 \mathrm{mg} / \mathrm{dl}$, glucosa $8 \mathrm{mg} / \mathrm{dl}$, leucocitos 1.000 céls $/ \mathrm{mm}^{3}$ (70\% PMN-30\% MN), tinción de Gram sin bacterias. Las baciloscopias, tinción Kinyoun y tinta china, RPC GeneXpert ${ }^{\circledR}$ TBC, RPC para CMV y VHS 1-2, VDRL, cultivo aeróbico, de hongos y Nocardia y panel molecular FilmArray ${ }^{\circledR}$ meníngeo fueron todos negativos. El nivel de galactomanano en plasma fue negativo y de $\beta$-D-glucano fue $284,1 \mathrm{pg} / \mathrm{ml}$ (positivo $\geq 80$ ). La ecocardiografía transtorácica no demostró vegetaciones.

$\mathrm{Al}$ cuarto día de tratamiento antimicrobiano el paciente persistía febril, con una leucocitosis de 14.550 céls $/ \mathrm{mm}^{3}$ ( $86 \%$ PMN) y PCR $313 \mathrm{mg} / \mathrm{l}$. Por ello y en contexto de un $\beta$-D-glucano elevado se tomaron nuevamente cultivos y se cambió el esquema antimicrobiano a meropenem i.v. $2 \mathrm{~g}$ cada $8 \mathrm{~h}$ y voriconazol i.v. $400 \mathrm{mg}$ cada $12 \mathrm{~h}$ el primer día y luego $200 \mathrm{mg}$ cada $12 \mathrm{~h}$. Todos los cultivos, incluyendo los hemocultivos fueron negativos. La segunda muestra de $\beta$-D-glucano fue negativa $(<31 \mathrm{pg} / \mathrm{ml})$.

Debido al tamaño y profundidad de la colección el equipo de neurocirugía inicialmente desestimó su drenaje quirúrgico; sin embargo, al día 11 desde inicio de los antimicrobianos, el paciente reinició fiebre, por lo que se realizó una RM de cerebro que mostró una progresión del absceso supra-tentorial derecho con extensión al sistema ventricular (Figura 3).

Dado los hallazgos imagenológicos y la mala evolución clínica se realizó un drenaje de la colección ventricular dando salida a 8 cc de LCR purulento. Se instaló un drenaje ventricular externo (DVE) frontal derecho y se inició la administración de vancomicina y amikacina 


\section{Caso Clínico}

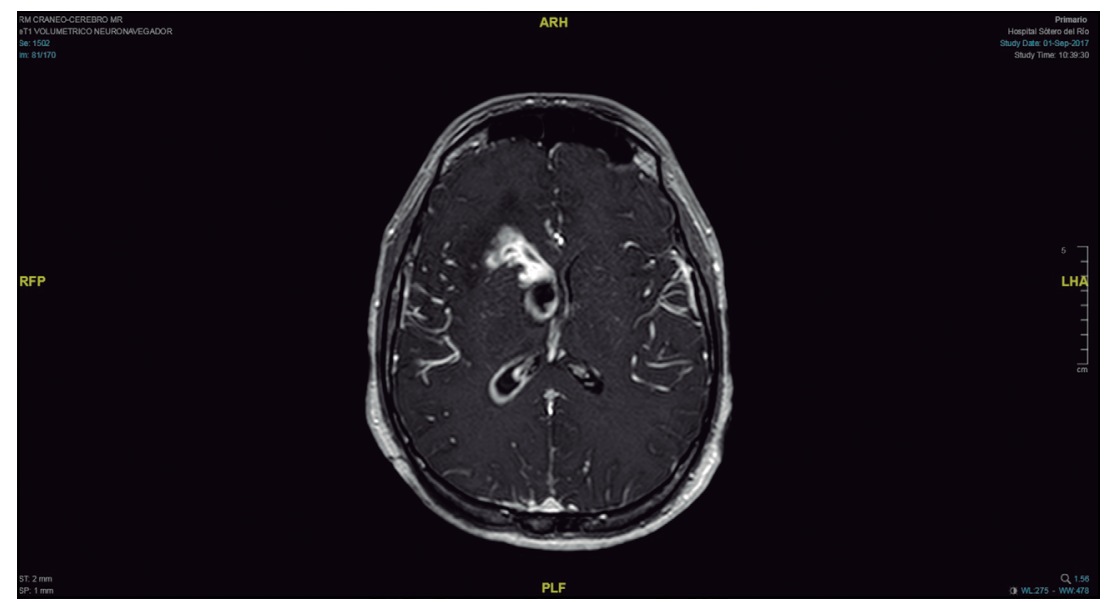

intraventricular. El estudio del LCR mostró células incontables, tinción de Gram con leucocitos abundantes, $\sin$ bacterias. La baciloscopia y cultivo fueron negativos, con ADA 14,1 U/1.

El paciente evolucionó con una neumonía asociada a VMI por Acinetobacter baumannii multiresistente solo sensible a colistín, por lo que se agregó colistín i.v. y en nebulizaciones. Posterior a este evento su evolución fue favorable, con descenso de la fiebre y de los parámetros inflamatorios, extubándose a las tres semanas de su ingreso.

Luego de un mes de hospitalización y de 12 días postdrenaje ventricular se informó una RPC universal en base de secuenciación proveniente de la colección, realizado en el Laboratorio de Biología Molecular del Hospital Clínico de la Universidad Católica, que fue negativa para hongos y positiva para $E$. corrodens. Se decidió suspender vancomicina (23 días de tratamiento), meropenem (19 días), voriconazol (16 días), vancomicina y amikacina intraventricular (9 días) y se reinició ceftriaxona 2 g cada $12 \mathrm{~h}$.

Al cuarto día del reinicio de ceftriaxona (día 27 de tratamiento antibiótico), el paciente se hizo afebril, sin déficit neurológico, con PCR en descenso (12 mg/l) y LCR normal. Desde el sexto día de ceftriaxona normalizó la PCR $(<5 \mathrm{mg} / \mathrm{l})$ en forma sostenida.

Considerando la bacteria aislada, se realizó una evaluación maxilo-facial que demostró caries en las piezas dentarias 3 y 15 . Se realizó además una ecocardiografía transesofágica (día 40 de hospitalización) que evidenció una vegetación 2 x $1 \mathrm{~mm}$ en el velo anterior de la válvula mitral. Tres semanas después, en una ecocardiografía transesofágica de control, no se observaron vegetaciones.

El día 63 de hospitalización se realizó una cisternostomía al tercer ventriculo y retiro del DVE; sin embargo, por reaparición de hidrocefalia se reinstaló un DVE 48 h después. Ocho días después (73 días de hospitalización) se instaló una derivación ventriculo peritoneal (DVP) permanente. El LCR de control tomado en este último procedimiento fue normal, con cultivos negativos. El paciente fue dado de alta luego de 84 días de hospitalización y habiendo completado 62 días de ceftriaxona desde su reinicio. La RM de cerebro confirmó la desaparición de las coleccciones (Figura 4).

A los siete meses el paciente necesitó un recambio de la DVP por disfunción valvular, sin evidencia clínica, de laboratorio o de imágenes de absceso cerebral, ventriculitis ni endocarditis.

\section{Discusión}

El presente caso clínico de un absceso cerebral y endocarditis por E. corrodens, según nuestro conocimiento, es el primero publicado en Chile. En el mundo se han

Figura 3. RM de cerebro día 11 de tratamiento antibacteriano, absceso cerebral fronto-parietal con vaciamiento ventricular. Nótese el reforzamiento de las paredes ventriculares con el uso de gadolinio (eT1 volumen neuronavegador).

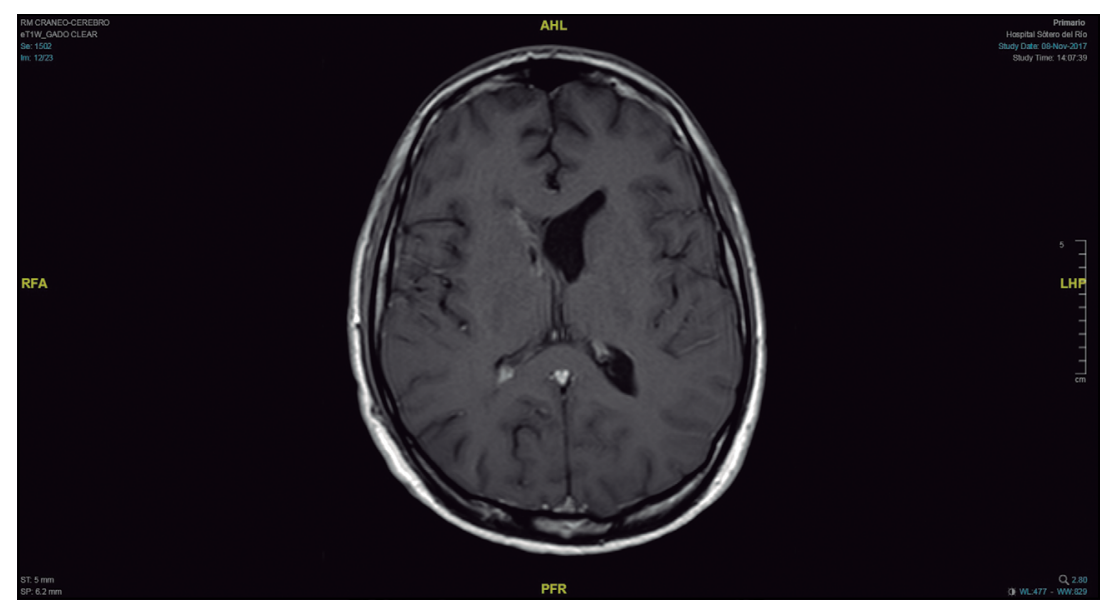

Figura 4. RM de cerebro día 84. Desaparición del absceso y pioventrículo al alta (eT1W gadolineo).

descrito, desde 1970 hasta la fecha, aproximadamente 34 casos de compromiso del sistema nervioso central (SNC) con formación de abscesos por E. corrodens $^{3-28}$ (Tabla 1).

Eikenella corrodens fue inicialmente denominada Bacillus corroding por Henriksen en 1948 debido a dos características: producir una depresión en medio del cultivo y ser un microorganismo anaerobio de crecimiento lento ${ }^{29}$. Eiken en 1958 lo describió como un bacilo gramnegativo anaerobio facultativo aislado de abscesos humanos (incluyendo cerebrales) y lo denominó Bacteroides corrodens ${ }^{30}$. Posteriormente, se determinó que esta bacteria presentaba una proporción de guanina-citosina diferente al género Bacteroides y que se desarrollaba en aerobiosis en medios de cultivo con hemina, por lo que se clasificó definitivamente en el año 1972 dentro de la 


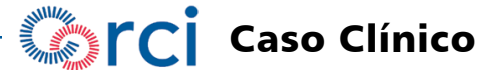

\begin{tabular}{|c|c|c|c|c|c|}
\hline Fuente & Edad / Sexo & Ubicación SNC & Factor de riesgo & Co-infección & Evolución \\
\hline \multirow[t]{2}{*}{ Marsden $^{3}$} & 9/F & Extra y subdural & Sinusitis & No & Fallece \\
\hline & $14 / \mathrm{F}$ & Frontal & Sinusitis & Streptococcus & Fallece \\
\hline \multirow[t]{2}{*}{ Kaplan ${ }^{4}$} & 9/M & Cerebeloso & Otitis media y mastoiditis & $\begin{array}{l}\text { Streptococcus } \\
\text { SCN* }^{*}\end{array}$ & Recuperado \\
\hline & $6 \mathrm{~m} / \mathrm{M}$ & Meningitis inicial & Neumonía y empiema & No & Recuperado \\
\hline Dorff $^{5}$ & 66/F & Meningitis inicial & Otitis media & No & Recuperado \\
\hline \multirow[t]{3}{*}{ Brooks $^{6}$} & $14 / \mathrm{F}$ & ND & Sinusitis & Polimicrobiano & Fallece \\
\hline & 9/M & ND & Otitis media & No & Fallece \\
\hline & $0,5 / \mathrm{M}$ & Meningitis inicial & Síndrome de Down & No & Desconocido \\
\hline Giassman ${ }^{7}$ & $33 / \mathrm{F}$ & Parietal & $\begin{array}{l}\text { Dental } \\
\text { Cardiopatía congénita }\end{array}$ & Streptococcus & Fallece \\
\hline \multirow[t]{2}{*}{ Rubenstein $^{8}$} & 14/M & Subdural & Sinusitis & S viridans & Recuperado \\
\hline & $15 / M$ & Cerebeloso & Trauma dental & Saureus & Recuperado \\
\hline Dudley ${ }^{9}$ & $12 / \mathrm{F}$ & Subdural & Sinusitis & Polimicrobiano & Recuperado \\
\hline Emmerson ${ }^{10}$ & $60 / F$ & Cerebeloso & Otitis media y mastoidectomía & Streptococcus spp. & Fallece \\
\hline Ingham"11 & $34 / \mathrm{M}$ & Frontal & Dental & S. milleri & Recuperado \\
\hline Sinkovic ${ }^{12}$ & 70/M & Temporal & Carcinoma seno maxilar & No & Recuperado \\
\hline Akhter $^{13}$ & $13 / \mathrm{M}$ & Epidural & Trauma orbitario y sinusitis & No & Recuperado \\
\hline Brill|14 & 71/M & Subdural & Sinusitis & S. viridans & Fallece \\
\hline Burdick $^{15}$ & $38 / \mathrm{M}$ & Parietal & No & No & Recuperado \\
\hline Porphyris $^{16}$ & $55 / \mathrm{M}$ & Cerebeloso & Otitis media & No & Recuperado \\
\hline Cheng ${ }^{17}$ & $67 / \mathrm{F}$ & Occipital & ND & No & ND \\
\hline Swanston ${ }^{18}$ & $3 / \mathrm{F}$ & ND & Tetralogia de Fallot & No & Recuperada \\
\hline Carola $^{19}$ & 35/M & Fronto-parietal & Abscesos dentales y MAV pulmonar & No & Recuperado \\
\hline Assefa ${ }^{20}$ & 10/M & Trombosis del seno cavernoso & $\begin{array}{l}\text { Sinusitis } \\
\text { Cardiopatía congénita }\end{array}$ & S. aureus & Hemiparesia \\
\hline \multirow[t]{2}{*}{ Sane $e^{21}$} & $2 / \mathrm{M}$ & Parieto-occipital y frontal & Cuerpo extraño en pulmón (CEP) & $\begin{array}{l}\text { S. } \alpha \text {-hemolyticus } \\
\text { S. aureus }\end{array}$ & Recuperado \\
\hline & $2 / \mathrm{M}$ & Temporal & CEP & S. $\alpha$-hemolyticus & Recuperado \\
\hline Asensi V22 & $54 / \mathrm{M}$ & Frontal & Exceso de limpieza dental & No & Recuperado \\
\hline Pogessi ${ }^{23}$ & $61 / \mathrm{M}$ & ND & Infección por VIH & No & ND \\
\hline Karunakaran ${ }^{24}$ & $2 / \mathrm{M}$ & Parietal bilateral & Cardiopatía congénita & S. milleri & Recuperado \\
\hline Gonzalez-Mera ${ }^{25}$ & & Absceso cerebral & Tumor carcinoide intestinal & S. intermedius & \\
\hline Pellachia ${ }^{26}$ & $57 / \mathrm{F}$ & Absceso cerebral & Diabetes, nucormicosis y aspergillosis pansinusal y orbital & No & Fallece \\
\hline Ozüm U27 & $41 / \mathrm{M}$ & Parietal & Infección periodontal crónica & $\begin{array}{l}\text { Entamoeba spp. } \\
\text { Prevotella spp. }\end{array}$ & Recuperada \\
\hline Fernández ${ }^{28}$ & 10/F & Intraventricular & Cardiopatía congénita cianótica & No & Recuperada \\
\hline
\end{tabular}


familia Neisseriaceae como un nuevo género llamado Eikenella, especie Eikenella corrodens ${ }^{1}$. Esta bacteria corresponde a un bacilo gramnegativo anaeróbico facultativo fastidioso, perteneciente al grupo HACEK, que forma parte de la microbiota de la orofaringe y tracto respiratorio superior. Crece lentamente en condiciones aeróbicas en agar sangre o agar chocolate y la presencia de $\mathrm{CO} 2$ al $5 \%$ favorece su desarrollo ${ }^{1}$.

Se ha catalogado como un microorganismo oportunista causante de infecciones principalmente de cabeza y cuello; se asocia especialmente pero no exclusivamente a neoplasias de esta zona. Son factores de riesgo reconocidos para la formación de un absceso cerebral por E. corrodens, la presencia de sinusitis, mastoiditis u otitis previa o conco-

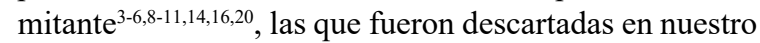
paciente, malformaciones cardíacas en niños ${ }^{6,7,18,20,24,29}$, infecciones odontológicas crónicas ${ }^{7,8,11,19,22,27}$ y diversas otras causas no claramente relacionadas tales como, la presencia de neoplasias ${ }^{12,25}$ cuerpos extraños en la vía aérea $^{21}$, diabetes mellitus ${ }^{26}$ e infección por $\mathrm{VIH}^{23}$. Cerca de $6 \%$ de los casos no se encuentra un factor favorecedor de la infección ${ }^{15,17}$ (Tabla 1).

Los mecanismos por los cuales este microorganismo accede al SNC son principalmente por contigüidad a infecciones locales (sinusitis y otitis), y hematógena en el caso de un origen endocárdico u odontogénico. Cuando los abscesos cerebrales son de origen odontogénico independientemente del microorganismo involucrado, suelen ser secundarios a caries en $60 \%$ de los casos $\mathrm{y}$ periodontitis en $43 \%{ }^{30}$. Incluso se ha demostrado la presencia de este microorganismo en la placa dentaria en casi $60 \%$ de personas sanas ${ }^{31}$. Está descrito el caso de un policía español obsesionado con su aseo bucal, que se provocaba frecuentemente sangramiento gingival causado por una enérgica limpieza dentaria, y que desarrolló un absceso cerebral frontal por E. corrodens ${ }^{22}$.

En el caso de nuestro paciente se asumió que el foco original pudo ser odontogénico o cutáneo para posteriormente infectar las válvulas cardíacas y por vía hematógena acceder al SNC. No había antecedentes de mordeduras humanas o traumatismos orales o faciales.

La presentación clínica de los abscesos cerebrales clásicamente se describe con una triada inespecífica de cefalea, fiebre y déficit neurológico focal que puede estar presente en $60 \%$ de los pacientes y que es variable de acuerdo a la ubicación y al tamaño del absceso ${ }^{32}$. Sin embargo, en ocasiones no es fácil determinar con certeza de que se trata de un proceso infeccioso y el diagnóstico diferencial debe realizarse principalmente con las neoplasias en SNC y enfermedades desmielinizantes, en particular con esclerosis múltiple, cuyas lesiones en la RM pueden tener características inflamatorias con captación de gadolinio, de modo similar a un absceso ${ }^{33}$.

Encontrar el agente etiológico involucrado en un absceso cerebral es complejo. La demora en el drenaje de colecciones y la exposición previa a antimicrobianos disminuyen ostensiblemente la posibilidad de aislar el microorganismo en los cultivos corrientes. En nuestro paciente, el panel molecular FilmArray ${ }^{\circledR}$ meníngeo en LCR obtenido por punción lumbar fue negativo, haciendo muy poco probable la presencia de los 14 patógenos que determina este $\mathrm{kit}^{34}$. El diagnóstico definitivo se logró una vez que se pudo obtener una muestra de LCR ventricular por técnica de RPC universal bacteriana con secuenciación, que estableció finalmente la presencia de E. corrodens.

La intervención neuro-quirúrgica ocurrió en relación al deterioro clínico, progresión del proceso inflamatorioinfeccioso y compromiso ventricular demostrado por una RM de control, 11 días después de inicio de tratamiento antibacteriano. La razón de esta demora se debió en un principio a la sospecha errónea de que se trataba de una neoplasia del SNC y en segundo lugar a la buena evolución inicial del paciente con tratamiento médico, en el contexto de un absceso cerebral de $3 \mathrm{~cm}$ de diámetro, considerado el tamaño máximo permitido para intentar un tratamiento conservador ${ }^{35-37}$. Sin embargo, ante la posible presencia de un pioventrículo, descrito con una mortalidad superior a $80 \%{ }^{38}$, se decidió intervenir, confirmándose esta complicación y realizando el drenaje del mismo.

Por otro lado, dentro de la etiología de las endocarditis infecciosas, alrededor de $3 \%$ corresponden al grupo HACEK. Dentro de este grupo, E. corrodens es la más infrecuente y ocurre generalmente en pacientes con factores de riesgo (uso de drogas intravenosas, daño valvular previo e inmunosupresión) ${ }^{2}$.

Respecto a la susceptibilidad antibacteriana de este agente suele ser sensible a ampicilina, ureidopenicilinas, cefalosporinas de segunda y tercera generación, tetracilinas, fluoroquinolonas, meropenem, imipenem y azitromicina, y resistente a clindamicina, eritromicina, metronidazol y aminoglucósidos ${ }^{1}$. Al momento de decidir el manejo antimicrobiano, se debe considerar que las infecciones de SNC por E. corrodens suelen ser polimicrobianas hasta en $50 \%$ de los casos, destacando especialmente la co-infección con Streptococcus milleri o viridans y con Staphylococus $\operatorname{spp}^{3,4,6-11,14,20,21,24,25,27}$.

En infecciones no graves se recomienda el uso de ampicilina, ampicilina/sulbactam o ácido clavulánico. En cambio, para infecciones graves como abscesos cerebrales o endocarditis se sugiere tratamiento con ceftriaxona en altas dosis no menor a $4 \mathrm{~g}$ al día o cefotaxima $2 \mathrm{~g}$ cada $6 \mathrm{~h}$ asociado a metronidazol i.v. $500 \mathrm{mg}$ cada $6 \mathrm{~h}$, dada la dificultad de cultivar agentes anaerobios y que cerca de un tercio de los abscesos cerebrales son polimicrobianos ${ }^{38,39}$.

El tratamiento antibacteriano de un absceso cerebral se debe prolongar por seis a ocho semanas y puede variar 


\section{- Grci caso clinico}

según su tamaño y la posibilidad de que se pueda drenar precozmente $^{38}$. La duración de la terapia antimicrobiana que recibió nuestro paciente (86 días) excede a esta referencia y se debió a la dificultad del diagnóstico etiológico y a las otras infecciones asociadas a la atención de salud que presentó durante su hospitalización.

La tasa de letalidad por absceso cerebral en una serie clínica chilena fue de $10 \%{ }^{40}$. En nuestra revisión, de los causados por $E$. corrodens fue mayor a $23 \% 0^{3,6,7,10,14}$. No se reconoce un factor de riesgo para este peor pronóstico y esto se puede explicar por lo poco frecuente de este agente infeccioso. Parece claro que las condiciones de inmunosupresión se asocian a un peor pronóstico y mortalidad.

\section{Referencias bibliográficas}

1.- Muihlhauser M. Eikenella corrodens. Rev Chilena Infectol. 2013; 30 (2): 163-4. doi: $10.4067 / \mathrm{S} 0716-10182013000200007$.

2.- Revest M, Egmann G, Cattoir V, Tattevin P. HACEK endocarditis: state-of-the-art. Expert Rev Anti Infect Ther. 2016; 14: 523-30. doi: $10.1586 / 14787210.2016 .1164032$.

3.- Marsden H B, Hyde W A. Isolation of Bacteroides corrodens from infection in children. J Clin Pathol. 1971; 24:117-9. doi: $10.1136 /$ jcp.24.2.117.

4.- Kaplan J M, McCraken Jr G H, Nelson J D. Infections in children caused by the HB group of bacteria. J Pediatr. 1973; 82: 398-403. doi: 10.1016/s0022-3476(73)80112-x.

5.- Dorff G J, Jackson L J, Rytel M W. Infections with Eikenella corrodens. A newly recognized human pathogen. Ann Intern Med. 1974; 80: 305-9. doi: 10.7326/0003-4819-80-3-305.

6.- Brooks G F, O’Donoghue J M, Rissing J P, Soapes K, Smith J W. Eikenella corrodens, a recently recognized pathogen: infections in medical-surgical patients and in association with methylphenidate abuse. Medicine (Baltimore) 1974; 53: 325-42.

7.- Glassman A B, Simpson J S. Eikenella corrodens: a clinical problem. J Am Dent Assoc. 1975; 91: 1237-41. doi: 10.14219/jada. archive.1975.0540.

8.- Rubenstein J E, Lieberman M F, Gadoth N. Central nervous system infection with Eikenella corrodens: report of two cases. Pediatrics 1976; 57: 264-5.

9.- Dudley J P, Goldstein E J C, George W L. Sinus infections due to Eikenella corrodens. Arch Otolaryngol. 1978; 104: 462-3.

10.- Emmerson A M, Mills F. Recurrent meningitis and brain abscess caused by Eikenella corrodens. Postgrad Med J. 1978; 54: 343-5. doi: 10.1136/pgmj.54.631.343.

11.- Ingham H R, Kalbag R M, Tharagonnet D, High A S, Sengupta R P, Selkon J B. Abscesses of the frontal lobe of the brain secondary to covert dental sepsis. Lancet 1978 ; 2: 497-9. doi: 10.1016/s0140-6736(78)92220-1.

12.- Sinkovics J, Plager C, Mills K. Eikenella corrodens as pathogen. Ann Intern Med. 1979; 90: 991. doi: 10.7326/0003-4819-90-6-991_1.
13.- Akhtar M J, Chandler J R. Periorbital, subgaleal and epidural empyemas secondary to Eikenella sinusitis. Ear Nose Throat J 1979; 58: 358-61.

14.- Brill C B, Pearlstein L S, Kaplan M, Mancall E L. CNS infections caused by Eikenella corrodens. Arch Neurol 1982; 39: 431-2. doi:10.1001/archneur.1982.00510190049015.

15.- Burdick C O, Erasmus D, Jayaram A, Schield P N, Kosch W. Eikenella brain abscess. JAMA 1982; 248: 1972-3. doi:10.1001/ jama.1982.03330160024010.

16.- Porphyris H. Central nervous system infections due to Eikenella corrodens. Surg Neurol. 1983; 19: 150-3. doi: 10.1016/0090-3019(83)904135.

17.- Cheng A F, South J R, French G L. Eikenella corrodens as a cause of brain abscess. Scand J Infect Dis. 1988; 20: 667-71. doi: $10.3109 / 00365548809035668$.

18.- Swanston W H, Cameron E S, Ramchander V. Eikenella corrodens brain abscess in a child with congenital heart disease. West Indian Med J. 1988; 37: 243-5.

19.- Caroli M, Arienta C. Rampini P M, Balbi S. Recurrence of brain abscess associated with asymptomatic arteriovenous malformation of the lung. Neurochirurgia (Stuttg) 1992; 35 : 167-70. doi: 10.1055/s-2008-1052272.

20.- Assefa D, Dalitz E, Handrick W, Lietz R, Braun W, Michalski H. Septic cavernous sinus thrombosis following infection of ethmoidal and maxillary sinuses: a case report. Int $\mathbf{J}$ Pediatr Otorhinolaryngol. 1994; 29: 249-55. doi: 10.1016/0165-5876(94)90171-6.

21.- Sane S M, Faerber E N, Belani K K. Respiratory foreign bodies and Eikenella corrodens brain abscess in two children. Pediatr Radiol. 1999; 29: 327-30. doi: 10.1007/ s002470050600.

22.- Asensi V, Alvarez M, Carton J A, Lago M, Maradona J A, Asensi J M, et al. Eikenella corrodens brain abscess after repeated periodontal manipulations cured with imipenem and neurosurgery. Infection 2002; 30: 240-2. doi: 10.1007/s15010-002-2004-6.

23.- Poggesi L, Scheggi V, Ammannati F, Bono P. A case of brain abscess by Eikenella corrodens in a HIV-positive patient. Ann Ital Med Int. 2003; 18: $162-6$.

24.- Karunakaran R, Marret M J, Hassan H,
Puthucheary S D. Eikenella corrodens from a brain abscess. Malays J Pathol. 2004; 26: 4952.

25.- González Mera L, Fernández González S, Martínez Yélamos S, Fernández Viladrich P. Brain abscess caused by Eikenella corrodens and Streptococcus intermedius associated with bowel carcinoid tumour. Med Clin. (Barc) 2005; 125: 117-8. doi:10.1157/13076739.

26.- Pellacchia V, Terenzi V, Moricca L M, Buonaccorsi S, Indrizzi E, Fini G. Brain abscess by mycotic and bacterial infection in a diabetic patient: clinical report and review of literature. J Craniofac Surg. 2006; 17: 578-84. doi: 10.1097/00001665-200605000-00034.

27.- Ozüm U, Karadağ O, Eğilmez R, Engin A, Oztoprak I, Ozçelik S. A case of brain abscess due to Entamoeba species, Eikenella corrodens and Prevotella species. Br J Neurosurg. 2008; 22: 596-8. doi: 10.1080/02688690801894646.

28.- Barrio Fernández P, Pérez Suárez J, MessingJünger A M. Case-based update: primary intraventricular brain abscess in a 10-year-old child. Childs Nerv Syst. 2015; 31: 2235-8. doi: 10.1007/s00381-015-2856-6.

29.- Eiken M. Studies on an anaerobic, rodshaped, gram-negative microorganism: Bacteroides corrodens n. sp. Acta Pathol Microbiol Scand. 1958; 43: 404-16.

30.- Moazzam A, Rajagopal S, Sedghizadeh P, Zada G, Habibian M. Intracranial bacterial infections of oral origin. J Clin Neurosci. 2015; 22: 800-6. doi: 10.1016/j.jocn.2014.11.015.

31.- Goldstein E J, Tarenzi L A, Agyare E O, Berger $\mathrm{J}$ R. Prevalence of Eikenella corrodens in dental plaque. J Clin Microbiol. 1983; 17: 6369.

32.- Vargas L, Alvarado M, Suárez A. Absceso cerebral: diagnóstico, manejo, complicaciones y pronóstico. Rev Chil Neurocirugía 2018; 44: 60-8. doi: 10.4067/S0716-10182006000200007.

33.- Aljure V de J, Pulido-Arias E A, RodríguezMonroy J A, Rodríguez-Mateus M N, Ramos-Hernández J. Diagnóstico diferencial de lesiones cerebrales con realce en anillo en tomografía computarizada y resonancia magnética. Duazary 2016; 13: 149-58. doi: 10.21676/2389783X.1721.

34.- Biomerieux panel FilmArray para meningoencefalitis. https://www.biomerieux.es/ 


\section{Caso Clínico}

diagnostico-clinico/productos/filmarrayr-panelmeningitisencefalitis-me. Fecha de acceso: 6 de julio de 2019.

35.- Aras Y, Sabanci P A, Izgi N, Boyali O, Ozturk $\mathrm{O}$, Aydoseli A, et al. Surgery for pyogenic brain abscess over 30 years: Evaluation of the roles of aspiration and craniotomy. Turk Neurosurg. 2016; 26: 39-47. doi: 10.5137/1019-5149. JTN.15099-15.1

36.- Carpenter J, Stapleton S, Holliman R.
Retrospective analysis of 49 cases of brain abscess and review of the literature. Eur $\mathbf{J}$ Clin Microbiol Infect Dis. 2007; 26: 1-11. doi: 10.1007/s10096-006-0236-6.

37.- Hall W A, Truwit C L. The surgical management of infections involving the cerebrum. Neurosurgery 2008; 62 (Sup 2): 519 30. doi: $10.1227 / 01$.neu.0000316255.36726.5b.

38.- Miranda H A, Castellar-Leones S M, Elzain M A, Moscote-Salazar L R. Brain abscess: current management. J Neurosci Rural Pract. 2013; 4 (Supp1): S67-81. doi: 10.4103/09763147.116472 .

39.- Gilbert D, Chambers H, Eliopoulos G, Saag M, Pavia A, editors. The Sanford Guide to Antimicrobial Therapy. 2019.

40.- Fica C A, Bustos G P, Miranda C G. Absceso cerebral a propósito de una serie de 30 casos. Rev Chilena Infectol. 2006; 23: 140-9. doi: 10.4067/S0716-10182006000200007. 